Retrospective Evaluation

\title{
Bilateral Thoracic Splanchnic Nerve Radiofrequency Thermocoagulation for the Management of End-Stage Pancreatic Abdominal Cancer Pain
}

Dimitrios Papadopoulos, MD, Georgia Kostopanagiotou, MD, PhD, and Chrysanthi Batistaki, MD, PhD

From: 2nd Department of Anesthesiology, School of Medicine, University of Athens, "Attikon" Hospital, Athens, Greece.

Address Correspondence: Chrysanthi Batistaki, MD, PhD 2nd Department of Anesthesiology School of Medicine University of Athens "Attikon" Hospital Athens, Greece E-mail:

chrysabatistaki@yahoo.gr

Disclaimer: There was no external funding in the preparation of this manuscript.

Conflict of interest: None.

Manuscript received: 10-11-12 Revised manuscript received: 11-26-12

Accepted for publication: 12-20-12

Free full manuscript: www.painphysicianjournal.com
Background: Pancreatic cancer pain is often severe and refractory to conservative therapies. Several interventional techniques have been described for the management of end-stage pancreatic cancer pain, with variable results and complications.

Objectives: The aim of this study was to assess the efficacy of bilateral radiofrequency thermocoagulation of splanchnic nerves for pain relief, the consumption of opioids, and the quality of life in patients suffering from severe pain due to pancreatic malignancies.

Study Design: A retrospective observational design.

Setting: The study includes patients with end-stage pancreatic abdominal cancer pain, which is refractory to conservative treatment.

Methods: Thirty-five patients were studied. They were evaluated prior to and after the radiofrequency thermocoagulation of both splanchnic nerves under fluoroscopic guidance. The assessment included the pain intensity (Numeric Rating Scale $0-10$ ), quality of life (selfreported quality of life score 0 - 10), and 24-hour consumption of opioids with monthly follow-up visits until the end of life.

Results: Follow-up was completed 6 months after the intervention. The pain scores, quality of life, and consumption of opioids were significantly improved during the entire follow-up period. A slight deterioration was noticed during the fifth month because of malignancy progression. No complications that could be attributed to the technique were observed.

Limitations: The study was not prospective and does not have a control group with a different intervention for comparisons.

Conclusion: Radiofrequency thermocoagulation of both splanchnic nerves may offer a safe and effective technique for pain management and quality of life improvement in patients with end-stage pancreatic cancer towards the end of life.

Key words: Splanchnic nerves, radiofrequency thermocoagulation, pancreatic cancer, pain

Pain Physician 2013; 16:125-133
D high mortality that causes severe abdominal and/or back pain. The disease is often resistant to analgesics, opioids, and adjuvant medications (1-3). The quality of life is seriously affected in these patients, and pain management in the context of palliative care should be an early part of the overall therapeutic plan (4-6).

The blockade or ablation of thoracic splanchnic nerves and celiac ganglia plays a major role in the 
pain management of most upper abdominal disorders, particularly chronic pancreatitis and pancreatic malignancies $(4,7-13)$. Thoracic splanchnic nerves carry the majority of the nociceptive stimuli from the upper abdominal viscera, and they are composed of preganglionic fibers, arising from the anterolateral horn of the spinal cord bilaterally, together with the T5-T12 ventral spinal roots (9-13).

Radiofrequency thermocoagulation is a minimally invasive technique that is performed under local anesthesia and fluoroscopic guidance. It is a target-selective technique, mostly indicated for the management of nociceptive chronic pain that is resistant to conservative therapy $(14,15)$, but it can also be used for the management of certain neuropathic pain conditions characterized by limited distribution (15). However, it is not indicated in centralized pain conditions in cases of co-existing pathology inside the spinal canal or if there are serious co-existing diseases, including severe psychopathology (14-16). Radiofrequency thermocoagulation is reported to be a more predictable and safe technique compared to chemical neurolysis of splanchnic nerves or celiac plexus blockade for the management of abdominal pain (17-22), but the evidence is still limited and more studies are needed to prove its efficacy in treating various pain states. In particular, only a few studies examined the actual efficacy of radiofrequency thermocoagulation alone or against other methods of interventional pain management in pancreatic cancer cases.

\section{Objectives}

The aim of this study was to assess the efficacy of bilateral thoracic splanchnic nerve radiofrequency thermocoagulation in pain management and its impact on the quality of life of patients with intractable abdominal pain due to end-stage pancreatic cancer.

\section{Methods}

This observational retrospective study includes patients who were evaluated in a private pain clinic from 2009 to 2012. The institutional review board approved the retrospective publication of these data. The patients enrolled in the study suffered from intractable abdominal pain due to end-stage pancreatic cancer and had received systemic therapy with opioids (transdermal, nasal, or sublingual fentanyl) in addition to adjuvant drugs for at least one month until they were referred for interventional pain management. The patients were evaluated extensively prior to the intervention to determine their complete medical history, in addition to their medications received and previously received pain therapies. All patients treated had no contraindications for regional blockade (coagulation disorders, local infection at the puncture site, sepsis, or severe displacement of intra-abdominal structures).

Pain assessment was performed using the Numeric Rating Scale (NRS, $0-10$ ), in addition to the self-reported quality of life (rated via a 0 - 10 visual analogue scale, where 0 represented the worst quality and 10 the best quality of life) according to Hauser's and Walsh's (2008) review on quality of life assessment in patients with cancer (23). The pain scores were recorded before (initial assessment visit) and one hour after the intervention, as well as after one week, 2 weeks, and then every month. Quality of life scores, in addition to consumption of opioids (calculated as the mean 24-hour quantity of fentanyl received by the patient during a 3-day period), was assessed preoperatively and then every month postoperatively until the end of patients' lives.

The procedure was performed in all patients after obtaining written informed consent. The technique was undertaken using fluoroscopic guidance and under local anesthesia. The patients were placed in the prone position, with a pillow placed under the abdomen. The $\mathrm{C}$-arm was positioned on the side of the patient, perpendicular to the trunk, ipsilateral to the puncture site, and then it was moved to the opposite side in exactly the same way. All patients had an intravenous line in place, and monitoring consisted of blood pressure measurement, $\mathrm{SpO}_{2}$, and continuous ECG.

A strictly aseptic technique was used, with sterile preparation and drape of the patient's back. Beginning with the T11 vertebra, the inferior end plates were visualized as a single line in the posterior-anterior view, and then the right costovertebral angle was recognized around the middle of the body of the vertebra, leaving enough space below. If not, then the C-arm image intensifier was moved slightly caudal, until the costovertebral angle was projected more cephalad, near the middle height of the vertebra's body. Then, the C-arm was rotated $15^{\circ}$ obliquely to the right. A metal forceps guide was placed on the patient's body in order for its tip to project right on the lateral edge of the vertebral body, just below the costovertebral angle (Fig. 1). This was the skin entry point, which was not more than 4 $\mathrm{cm}$ from the spinous processes, to reduce the risk of pneumothorax. 


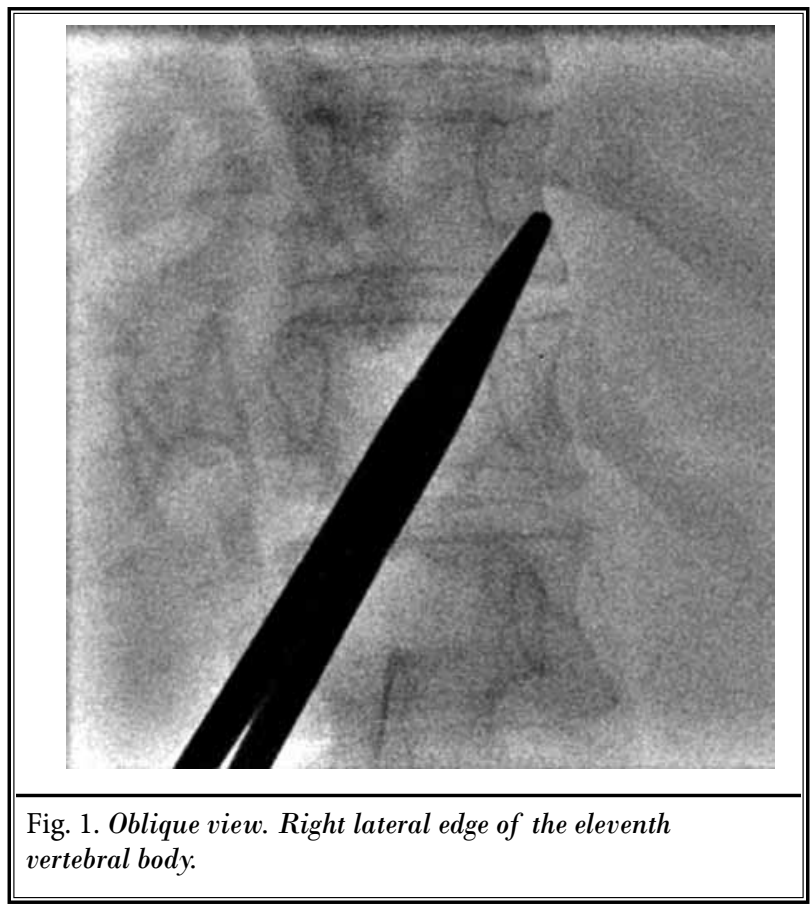

After local anesthesia with $3 \mathrm{~mL}$ of $1 \%$ lidocaine into the superficial tissues at the puncture site to a depth of $3-4 \mathrm{~cm}$, the introducer angiocath needle $(16 \mathrm{G})$ was inserted superficially and advanced under tunnel view fluoroscopic guidance, aiming to reach the lateral border of the vertebra. The introducer was then projected using the oblique view (Fig. 2). After stylet removal, the radiofrequency blunt curve needle (OWL-sterile single use cannula, $20 \mathrm{G}, 150 \mathrm{~mm}, 15 \mathrm{~mm}$ active tip; Diros, Canada) was introduced, with the needle tip facing the body of the vertebra. The needle was advanced slowly and carefully for another $1-2 \mathrm{~cm}$, staying approximately in contact with the vertebral bone. The depth of the needle was checked using a true lateral view and advanced further, still or approximately in contact with the periosteum, until the active tip reached the area at the border between the anterior and middle third of the vertebral body (Fig. 3). The correct final needle placement was confirmed by viewing a posterior-anterior image, where the needle tip was in touch with the lateral border of the vertebra, below the costovertebral angle (Fig. 4). The same procedure was repeated at the level of T12 vertebra. The stylets of the radiofrequency needles were removed, and the electrodes ( $\mathrm{TCH}-15 \mathrm{~S}, 20$ $\mathrm{G}$ reusable probe; Diros, Canada) were introduced in the needles and connected to the radiofrequency generator (OWL-URF-3AP; Diros, Canada) (Figs. 5 and 6).

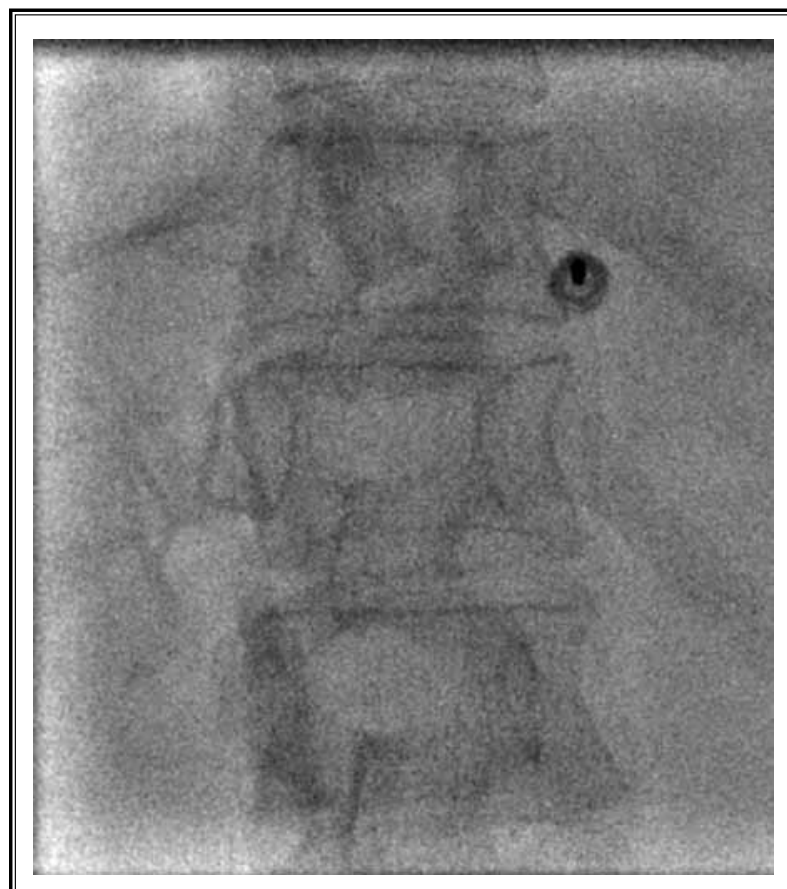

Fig. 2. Oblique view. Tunnel view technique. Introducer at the lateral edge of the vertebral body.

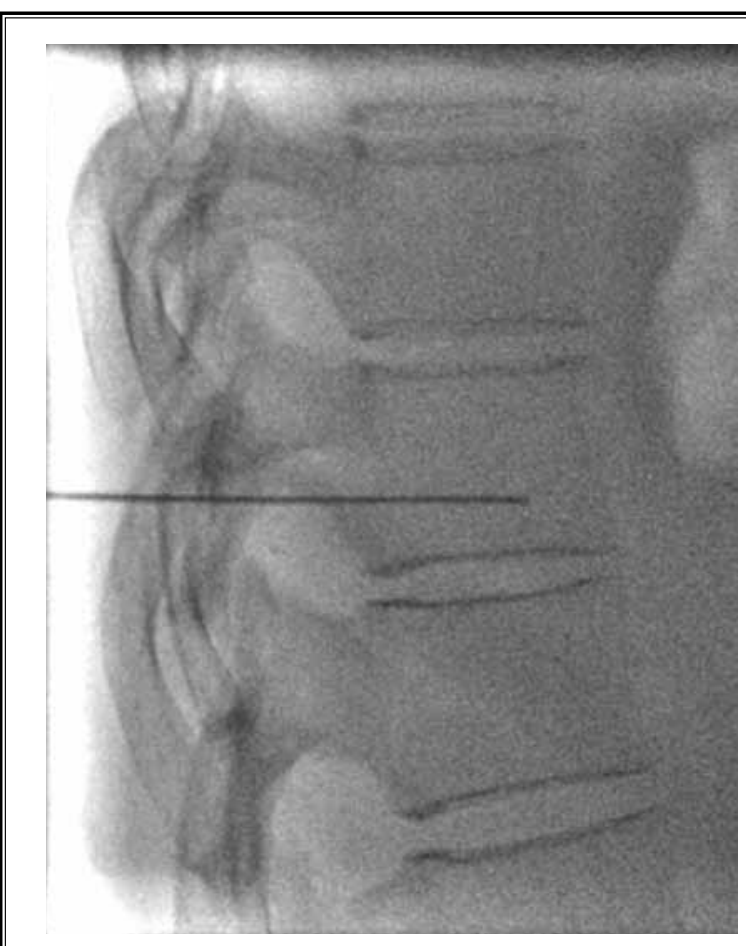

Fig. 3. Lateral view. Final radiofrequency needle position. 


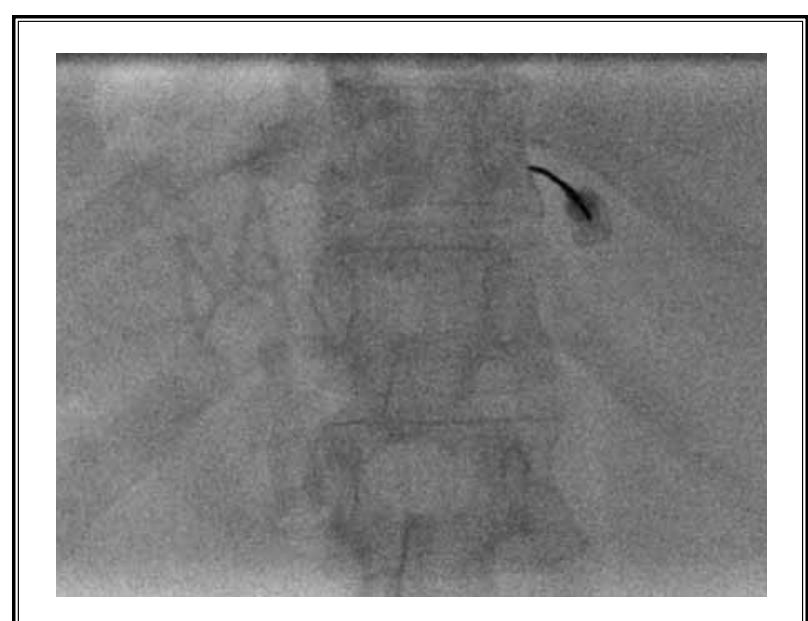

Fig. 4. Posterior-anterior view. Tip of the radiofrequency needle in touch with the lateral border of the vertebra.

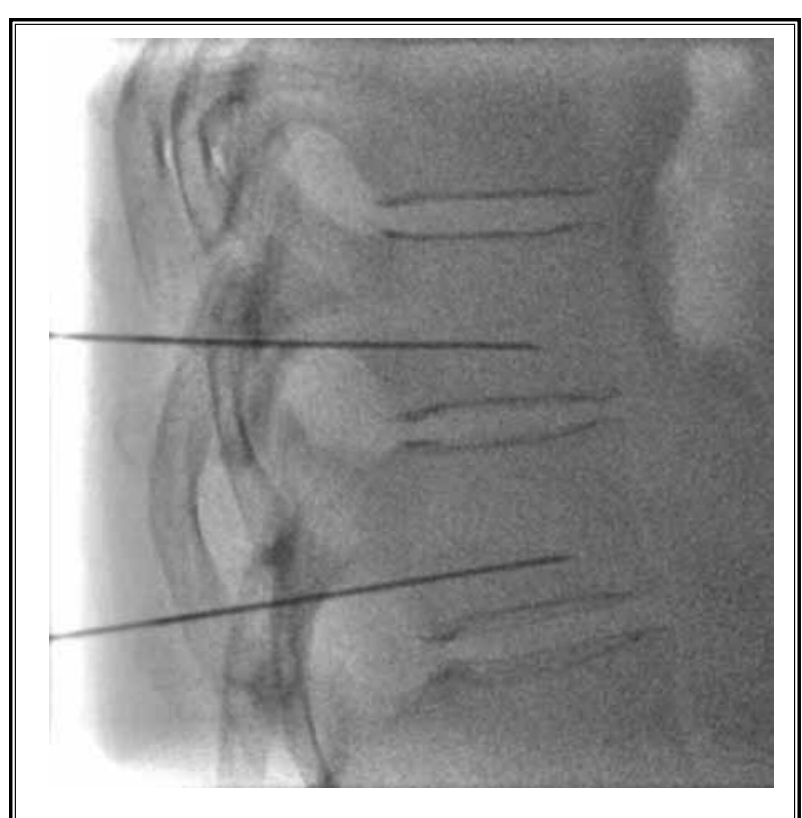

Fig. 5. Lateral view. Radiofrequency needles final position.

To identify the nerves, a sensory and motor test followed. The parameters for sensory stimulation were as follows: frequency $50 \mathrm{~Hz}$, pulse width one ms, and voltage up to $1.0 \mathrm{~V}$. The patient was expected to report pain, pressure, or general discomfort at the abdominal area, or sometimes at the lumbar region. If this did not occur, the needle was advanced a few millimeters further to the front, or withdrawn a few millimeters

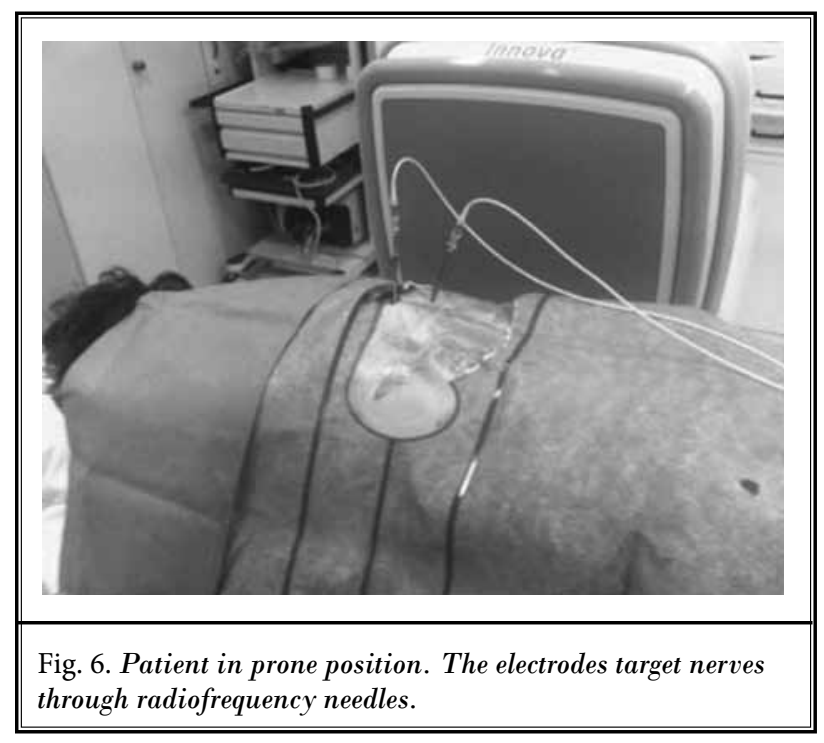

back, until proper sensory response was obtained. Motor stimulation settings were as follows: frequency 2 $\mathrm{Hz}$, pulse width $1 \mathrm{~ms}$, and voltage up to $2.5 \mathrm{~V}$. No contractions of the intercostal muscles should occur during the test, and if this happened (meaning that the active tip of the electrode was too close to the intercostal nerve), the electrode was advanced a few millimeters anteriorly. The lesion type included 2 monopolar radiofrequency lesions simultaneously at $85^{\circ} \mathrm{C}$, with a ramp time to a maximum temperature of $15 \mathrm{sec}$ and the total duration of the lesion, including ramp time, of $90 \mathrm{sec}$. Immediately after the generation of every lesion, 2.5 $\mathrm{mL}$ of a $10 \mathrm{~mL}$ solution, containing $2 \mathrm{ml}$ of dexamethasone $(4 \mathrm{mg} / \mathrm{mL})$ and $8 \mathrm{~mL}$ of ropivacaine $(7.5 \mathrm{mg} / \mathrm{mL})$, were injected to reduce the postoperative tissue edema and discomfort.

All patients were closely observed postoperatively for pain, sensory and motor deficits, as well as for vital signs. The patients were discharged from the clinic after obtaining a normal chest radiograph, normal vital signs assessed by medical personnel, and after a total period of 6 hours. All patients were advised to stay overnight close to a medical facility and not to travel by air during the next few days if not examined by their doctor, to minimize the risk of pneumothorax being misdiagnosed.

All patient data regarding pain, quality of life score, and opioids consumption were analyzed with the Statistical Package for Social Sciences (SPSS 13.0 for Windows), using non-parametric Wilcoxon matched pair analysis and with a statistical significance set as $P$ $<0.05$. 


\section{Results}

Thirty-five patients were studied, all suffering from pancreatic cancer pain, with a mean age of 67.8 years and a mean duration of pain of 3 months. Demographic characteristics of patients are presented in Table 1. No complications occurred intraoperatively, and all patients tolerated the procedure well, without experiencing any serious pain or discomfort that would require modification of the analgesic plan. The postoperative period was also uneventful. No significant side effects occurred that could be attributed to the interventional technique.

The follow-up period was up to 6 months postoperatively, as most patients died within 4 months postintervention (only 9 patients were alive at 4 months, 4 at 5 months, and none at 6 months). The mean NRS score $(0-10)$ prior to therapy was 8.9 (range $7-10)$. Pain was significantly reduced compared to baseline values in all patients immediately postoperatively, as well as throughout the entire follow-up period (Fig. 7) $(P<0.05)$, except from 5 months, at which point the reduction in pain scores was not statistically significant. The overall quality of life during the last months of life was significantly improved by the procedure (Fig. 8). The baseline quality of life score $(0-10)$ was as low as 1.05 (range $0-3$ ) but increased significantly $(P<0.05)$ for the next 4 months after therapy. However, during the last month of life, the quality of life was reported to be slightly worse than in previous months. Although the difference from baseline preoperative values was no longer statistically significant, the quality of life was still improved (Fig. 8).
Regarding opioids consumption, baseline daily consumption of fentanyl was 1611.43 (range 600 3000) $\mathrm{mcg} / 24$ hours and was significantly reduced during the first 4 postoperative months (Fig. 9) $(P<$ $0.05)$. At 5 months postoperation, a slight increase in opioid consumption was noted, but there was no statistical significance compared with baseline values. The adjuvant analgesics administered included corticosteroids, pregabalin, and amitriptyline in individualized doses.

The complications included temporary diarrhea in 11 patients and temporary lower abdominal pain due to intestinal colic in 5 patients. Both incidents lasted for $<7$ days postoperatively. No other complications that could be attributed to the technique were observed.

Table 1. Demographic characteristics, duration of procedure, overnight stay, and complications rate in patients treated with percutaneous radiofrequency thermocoagulation of splanchnic nerves.

\begin{tabular}{|l|c|}
\hline Age (years) & 67.8 (range 56-82) \\
\hline Gender (male/female) & $19 / 16$ \\
\hline Duration of procedure (min) & 65 (range 60-90) \\
\hline Overnight stay (n) & 0 \\
\hline $\begin{array}{l}\text { Complications (n) } \\
\text { Temporary diarrhea } \\
\text { Temporary lower abdominal pain due to } \\
\text { intestinal colic }\end{array}$ & 11 \\
\hline
\end{tabular}

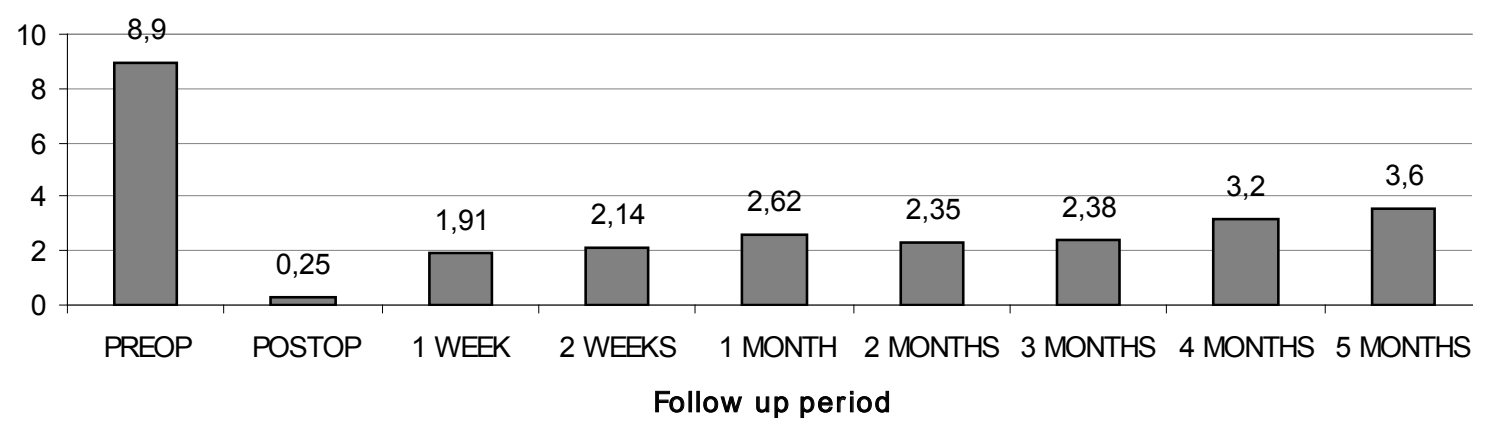

Fig. 7. Mean preoperative and postoperative values of Numeric Rating Scale (NRS 0-10) during the whole follow-up period. 


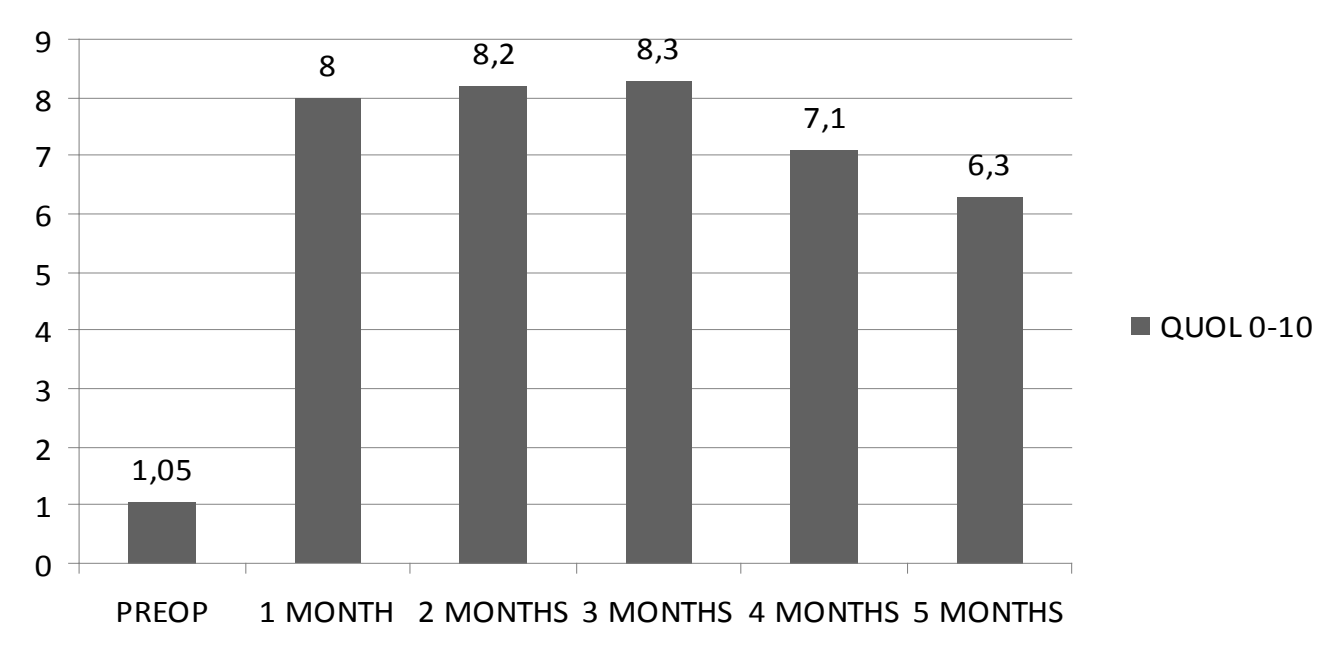

Fig. 8. Mean preoperative and postoperative values of Quality of Life score (QOL 0-10) during the whole follow-up period.

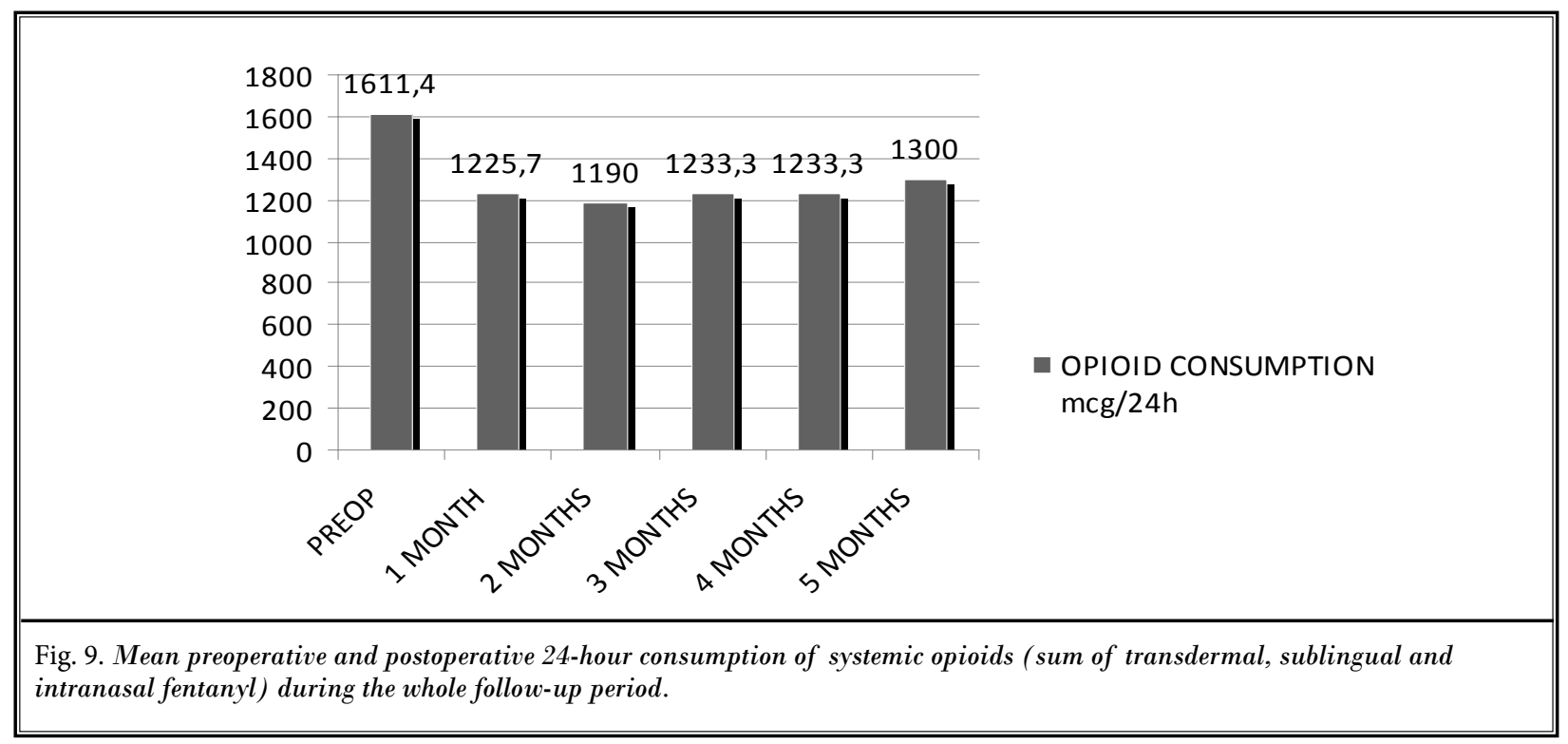

\section{Discussion}

Pancreatic cancer pain has a poor prognosis, regardless of advances in surgery, radiation, and chemotherapy. Therefore, palliative care and especially pain management remains an important issue for these patients $(5,24)$. Thoracic splanchnic nerves carry the majority of the nociceptive stimuli from the upper abdominal viscera. Thus, they are a perfect target for interrupting these pain signals because they are contained in a narrow compartment, which is defined medially by the vertebral bodies, laterally by the pleura, ventrally by the posterior mediastinum, and dorsally by the pleura attachment to the vertebra. This compartment is limited caudally by the crura of the diaphragm $(4,7,12,13)$. The thoracic splanchnic nerves are composed of preganglionic fibers, together with T5-T12 ventral spinal roots, which synapse on their way paravertebrally with the white communicating rami, before reaching the sympathetic chain, and pass through the sympathetic 
ganglia to finally synapse with the celiac ganglia. The great splanchnic nerve arises from the T5-T10 nerve roots. The lesser splanchnic nerve arises from the T10T11 nerve roots, and the least splanchnic nerve arises from the T11-T12 nerve roots bilaterally (9-13).

It has generally been reported that pain occurs in about $80-85 \%$ of patients with non-operable pancreatic malignancies, and most of the time conventional analgesics fail to offer sufficient pain relief $(4,24)$. This was the case for our patients, who suffered from refractory, abdominal pain (with initial NRS values as high as 8.9/10), but they succeeded in achieving a significant reduction of pain scores immediately after bilateral splanchnic nerve radiofrequency thermocoagulation, as well as during the entire follow-up period. At the 2-month and 3-month follow-up visits, almost all patients had an NRS score $<3$, which is considered an excellent result. The slight increase in the NRS values observed after 5 months was due to the progression of the disease, but the pain scores were still $<4 / 10$ and lower than the preoperative values.

Quality of life was also significantly improved during the follow-up period as reported by patients, and the consumption of opioids was reduced, except for at 5 months (where only 4 patients were still alive). This deterioration of quality of life during the last months of life has also been described by other authors performing palliative procedures for pain management, such as the study of Ozyalcin et al (25). The authors explained this deterioration as a result of tumor progression and paraneoplastic side effects.

Interventional pain management represents an attractive treatment option for patients with refractory abdominal pain due to pancreatic cancer and includes celiac plexus neurolysis (percutaneous, ultrasoundguided, endoscopic neurolysis), splanchnic nerve neurolysis, and splanchnic nerve radiofrequency thermocoagulation. Celiac plexus blockade is the most widely studied interventional technique for abdominal pain relief and has been evaluated in many studies (25-34). Efficacy is reported to be high for short- and long-term pain relief $(25,32,33)$ using the percutaneous technique but with some severe complications. However, chemical neurolysis of the celiac plexus does not always lead to adequate pain control, possibly due to degeneration and fibrosis of nerves, ganglia, and nerve-adjacent tissues from the injected chemical substances $(32,34)$. The main complications that accompany celiac plexus blockade/neurolysis are reported to be paralysis, pare- sis, paresthesia, pneumothorax, pleural empyema, diarrhea, hypotension, retroperitoneal fibrosis and abscess formation, gastric perforation, aortic pseudoaneurysm formation, and abdominal aortic dissection $(32,33)$. In a recent study by Yang et al (2012) (35), who performed a consecutive neurolysis of celiac plexus for the management of refractory abdominal pain in 12 patients, diarrhea and hypotension were reported quite often, but pain relief was significant for up to 6 months after the procedure. In contrast, Wiechowska-Kozłowska et al (2012) (32) used an endoscopic ultrasound-guided technique for celiac plexus blockade and succeeded in pain relief in $69 \%$ of patients treated, but $31 \%$ of the patients presented no improvement. The failure of response, according to the authors, suggests that perhaps the transmission of pain signals continues, as destruction of nerve fibers is only partial, and, in addition, there is regeneration and fibrosis over time.

Ozyalcin et al (25) conducted a randomized controlled trial testing the efficacy of celiac plexus versus splanchnic nerves neurolysis in patients with pancreatic cancer pain (19 and 20 patients in each group respectively) and revealed that splanchnic nerve neurolysis led to significantly better pain relief, quality of life, and analgesic consumption until the end of the patients' lives. The authors support the superiority of splanchnic nerves blockade in pancreatic cancer pain, mostly due to a superior analgesic effect, fewer complications (5 patients from the celiac group reported severe diarrhea and 2 required inotropic support for 24 hours after the intervention due to hemodynamic disturbances), and reduced analgesic consumption until the end of life. Similarly, Marra et al (1999) (34), in an older study comparing the 2 neurolytic methods, concluded that although they were both efficient in pain relief, splanchnic nerve blockade was superior in most cases. Similar findings regarding the efficacy of neurolysis of splanchnic nerves were observed in a study comparing chemical neurolysis with placebo in patients with histologically proven non-operable pancreatic cancer (35). Therefore, according to the current literature, the neurolysis of the splanchnic nerves appears to have a favorable profile in regards to pain relief and the complications compared to celiac plexus blockade. However, neurolysis itself is followed by serious side effects due to the unpredictable spread of alcohol or phenol during the procedure (19,31-33). This is the reason why it was followed up with the development of newer, more sophisticated interventional techniques, such as tho- 
racoscopic splanchnicectomy (36) and fluoroscopically guided radiofrequency ablation, which is the technique used in our study (37-43).

Radiofrequency thermocoagulation of the splanchnic nerves has not been widely tested for treatment of pancreatic cancer pain. It was initially developed by Raj et al in 1999 (41) and is based on the anatomic location of thoracic splanchnic nerves, which makes them the perfect target for selective radiofrequency ablation. One of the earliest studies performed, by Raj et al (42) in 107 patients with abdominal pain of malignant and non-malignant origin, revealed good to excellent results in $55-70 \%$ of patients for pain scores, but no data were mentioned regarding quality of life. The results from another study by Garcea et al (43), who treated 10 patients with non-malignant chronic pancreatic pain with radiofrequency ablation of splanchnic nerves, revealed that the technique led to a decrease in pain scores, consumption of opioids, and acute admissions for pain, in addition to improvement of other parameters (such as mood, general perception of health, everyday activity levels, and long-term perception of debilitating chronic pain) for a median follow-up period of 24 months. These findings are in accordance with our results, with patient outcomes presenting significant improvement in terms of pain intensity and quality of life. The side effects of therapy, such as diarrhea and intestinal colic, did occur, but they were only temporary (lasting $<7$ days) and did not affect the overall acceptance and efficacy of the technique. No cases of pneumothorax occurred in our patients, perhaps because the technique was performed using blunt, curved radiofrequency needles (41-44) (which prevent penetration of the lung) and because the skin entry point was not more than $4 \mathrm{~cm}$ from the spinous processes. However, clinical experience with the technique, as well as adequate information delivered to the patients regarding overnight stays close to a medical facility and examination by a clinician before air travel, are essential in avoiding such complications.

The retrospective basis of the study and the absence of a control group of patients with a different intervention are the main limitations of our results. However, due to the limited literature on the subject (especially on malignant pain management), reviewing the cases of 35 patients who underwent the procedure is very informative for evaluating the actual outcome of the technique in regard to malignant pancreatic pain management.

The evaluation of patients with pancreatic cancer should also include an assessment of the patients' quality of life (24). Evaluating every patient according to the principles of the biopsychosocial model is very important for achieving actual control of symptoms, support of the patient and family, and successful pain management (45). The best interventional technique to achieve this has yet to be identified, as there are an insufficient number of randomized, controlled trials examining pain management in this clinical setting.

\section{Conclusion}

Our results suggest that bilateral thoracic splanchnic nerve radiofrequency thermocoagulation significantly reduced pancreatic abdominal cancer pain, reduced consumption of systemic opioids, and improved patients' quality of life during the end-stage phase of their disease. Bilateral thoracic splanchnic nerves radiofrequency thermocoagulation may offer a minimally invasive, safe, and effective technique for the management of pancreatic abdominal cancer pain. Expertise with the technique and adequate information delivery to patients are essential for minimizing the risk of perioperative pneumothorax.

\section{References}

1. Ventrafridda V, Tamburini M, Caraceni A, De Conno F, Naldi F. A validation study of the WHO method for cancer pain relief. Cancer 1987; 59:850-856.

2. Graham AL, Andren-Sandberg A. Prospective evaluation of pain in exocrine pancreatic cancer. Digestion 1997; 58:542-549.

3. American Cancer Society. Cancer Facts \& Figures 2012. American Cancer Society, Atlanta, 2012.
4. Loukas M, Klaassen Z, Merbs W, Tubbs RS, Gielecki J, Zurada A. A review of the thoracic splanchnic nerves and celiac ganglia. Clin Anat 2010; 23:512-522.

5. Saif MW. Palliative care of pancreatic cancer. JOP 2011; 12:355-567.

6. Velanovich $\mathrm{V}$. The association of qualityof-life measures with malignancy and survival in patients with pancreatic pathology. Pancreas 2011; 40:1063-1069.

7. Boas RA. Sympathetic blocks in clinical practice. Int Anesthesiol Clin 1978;16:149.

8. Raj P, Rauck R, Racz G. Autonomic nerve blocks. In: Raj P (ed). Pain Medicine: A Comprehensive Review. Mosby, St Louis, MO, 1996, pp 227-255.

9. Gest TR, Hildebrandt $S$. The pattern of the thoracic splanchnic nerves as they pass through the diaphragm. Clin Anatomy 2010; 22:809-814.

10. Yang HJ, Gil YC, Lee WJ, Kim TJ, Lee HY. Anatomy of thoracic splanch- 
nic nerves for surgical resection. Clinl Anatomy 2008; 21:171-177.

11. Loukas M, Klaassen Z, Merbs W, Tubbs S, Gielecki J, Zurada A. A review of the thoracic splanchnic nerves and celiac ganglia. Clinl Anatomy 2010; 23:512-522.

12. Naidoo N, Partab P, Pather N, Moodley J, Singh B, Satyapal KS. Thoracic splanchnic nerves: Implications for splanchnic denervation. J Anatomy 2001; 199:585-590.

13. Waldman SD. The splanchnic nerves. In: Pain Review. Saunders Elsevier, Philadelphia, PA. 2009.

14. Van Boxem K, van Eerd M, Brinkhuize T, Patijn J, van Kleef M, van Zundert J. Radiofrequency and pulsed radiofrequency treatment of chronic pain syndromes. The available evidence. Pain Practice 2008; 8:385-393.

15. Rea W, Kapur S, Mutagi H. Radiofrequency therapies in chronic pain. Contin Educ Anaesth Crit Care Pain Adv 2011; 1:35-38.

16. Chua N, Vissers K, Sluijster M. Pulsed radiofrequency treatment in interventional pain management: Mechanisms and potential indications-- a review. Acta Neurochir 2011; 153:763-771.

17. Van Dongen RT, Crul BJ. Paraplegia following coeliac plexus block. Anaesthesia 1991; 46:862-863.

18. Gaudy JH, Tricot C, Sezeur A. Serious heart rate disorders following perioperative splanchnic nerve phenol nerve block. Can J Anaesth 1993; 40:357-359.

19. Lalanne B, Baubion O, Sezeur A, Tricot C, Gaudy JH. Circulatory arrest after splanchnic neurolysis with phenol in unresectable cancer of the pancreas. Ann Chir 1994; 48:1025-1028.

20. Rosenthal JA. Diaphragmatic paralysis complicating alcohol splanchnic nerve block. Anesth Analg 1998; 86:845-846.

21. Abdalla EK, Schell SR. Paraplegia following intraoperative celiac plexus injection. J Gastrointest Surg 1999; 3:668-671.

22. Kumar A, Tripathi SS, Dhar D, Bhattacharya A. A case of reversible paraparesis following celiac plexus block. Reg Anesth Pain Med 2001; 26:75-78.

23. Hauser K, Walsh D. Visual Analogue Scales and assessment of quality of life in patients with cancer. J Support Oncol 2008; 6:277-282.
24. Lee V, Cheng H, Li G, Saif MW. Quality of life in patients with pancreatic cancer. JOP 2012; 13:182-184.

25. Ozyalcin S, Talu GK, Camlica H, Erdine S. Efficacy of celiac plexus and splanchnic nerve blockades in body and tail located pancreatic cancer pain. Eur J Pain 2004; 8: 539-545.

26. Kawamata $M$, Ishitani $K$, Ishikawa $K$, Sasaki H, Ota K, Omote K, Namiki A. Comparison between celiac plexus block and morphine treatment on quality of life in patients with pancreatic cancer pain. Pain 1996; 64:597-602.

27. Mercadante S. Celiac plexus block vs analgesics in pancreatic cancer pain. Pain 1993; 52:187-192.

28. Polati E, Finco G, Gottin L, Bassi C, Pederzoli $P$, Ischia S. Prospective randomized double-blind trial of neurolytic coeliac plexus block in patients with pancreatic cancer. Br J Surg 1998; 85:199-201.

29. Rykowski JJ, Hilgier M. Efficacy of neurolytic celiac plexus block in varying locations of pancreatic cancer: Influence on pain relief Anesthesiology 2000; 92:347-354.

30. Vranken JH, Zuurmond WW, de Lange $J$ J. Increasing the efficacy of a celiac plexus block in patients with severe pancreatic cancer pain. J Pain Symptom Manage 2001; 22:966-977.

31. Wong GY, Schroeder DR, Carns PE, Wilson JL, Martin DP, Kinney MO, Mantilla CB, Warner DO. Effect of neurolytic celiac plexus block on pain relief, quality of life, and survival in patients with unresectable pancreatic cancer: A randomized controlled trial. JAMA 2004; 29:1092-1099.

32. Wiechowska-Kozlowska A, Boer K, Wojcicki M, Milkiewicz P. The efficacy and safety of endoscopic ultrasoundguided celiac plexus neurolysis for treatment of pain in patients with pancreatic cancer. Gastroenterol Res Pract 2012; 2012:503098.

33. Eisenberg E, Carr DB, Chalmers TC. Neurolytic celiac plexus block for treatment of cancer pain: A meta-analysis. Anesth Analg 1995; 80:290-295.

34. Marra V, Debernardi F, Frigerio A, Menna S, Musso L, Di Virgilio MR. Neurolytic block of the celiac plexus and splanchnic nerves with computed to- mography. The experience in 150 cases and an optimization of the technique. Radiol Med 1999; 98: 183-188.

35. Yang FR, Wu BS, Lai GH, Wang Q, Yang LQ, He MW, Ni JX. Assessment of consecutive neurolytic celiac plexus block (NCPB) technique outcomes in the management of refractory visceral cancer pain. Pain Med 2012; 13:518-521.

36. Baghdadi S, Abbas MH, Albouz F, Ammori BJ. Systematic review of the role of thoracoscopic splanchnicectomy in palliating the pain of patients with chronic pancreatitis. Surg Endosc 2008; 22:580-588.

37. Lillemoe KD, Cameron JL, Kaufman HS, Yeo C), Pitt HA, Sauter PK. Chemical splanchicectomy in patients with unresectable pancreatic cancer: A prospective randomized trial. Ann Surg 1993; 217:447-457.

38. Gardner AM, Solomou G. Relief of the pain of unresectable carcinoma of pancreas by chemical splanchnicectomy during laparotomy. Ann $R$ Coll Surg Engl 1984; 66:409-411.

39. Fields S. Retrocrural splanchnic nerve alcohol neurolysis with a CT-guided anterior transaortic approach. J Comput Assist Tomogr 1996; 20:157-160.

40. Noble M, Gress FG. Techniques and results of neurolysis for chronic pancreatitis and pancreatic cancer pain. Curr Gastroenterol Rep 2006; 8:99-103.

41. Raj PP, Thomas J, Heavner J, Racz G, Lou L, Day M, Shaw BC. The development of a technique for radiofrequency lesioning of splanchnic nerves. Curr Rev Pain 1999; 3:377-387.

42. Raj PP, Sahinler B, Lowe M. Radiofrequency lesioning of splanchnic nerves. Pain Pract 2002; 2:241-247.

43. Garcea G, Thomasset S, Berry D, Tordoff S. Percutaneous splanchnic nerve radiofrequency ablation for chronic abdominal pain. ANZ J Surg 2005; 75:640-644.

44. Finch PM, Racz GB, McDaniel K. A curved approach to nerve blocks and radio frequency lesioning. Pain Digest 1997; 7:251-257.

45. Bhaskar AK. Interventional management of cancer pain. Curr Opin Support Palliat Care 2012; 6:1-9. 
